ARTICLE

\title{
Orbital stability analysis and photometric characterization of the second Earth Trojan asteroid $2020 \mathrm{XL}_{5}$
}

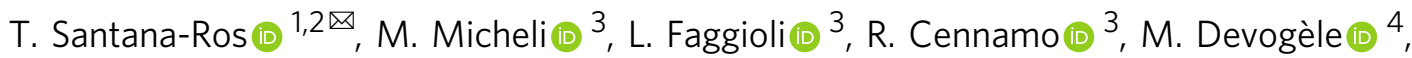
A. Alvarez-Candal (1) 5,6,7, D. Oszkiewicz (1) ${ }^{8}$, O. Ramírez ${ }^{9}$, P.-Y. Liu ${ }^{6}$, P. G. Benavidez (10 1,6, A. Campo Bagatin (1) ${ }^{1,6}$, E. J. Christensen ${ }^{10}$, R. J. Wainscoat (1) ${ }^{11}$, R. Weryk (1) ${ }^{12}$, L. Fraga (1) ${ }^{13}$, C. Briceño (1) ${ }^{14} \&$ L. Conversi (i) ${ }^{3,15}$

Trojan asteroids are small bodies orbiting around the $L_{4}$ or $L_{5}$ Lagrangian points of a Sunplanet system. Due to their peculiar orbits, they provide key constraints to the Solar System evolution models. Despite numerous dedicated observational efforts in the last decade, asteroid 2010 TK $_{7}$ has been the only known Earth Trojan thus far. Here we confirm that the recently discovered $2020 \mathrm{XL}_{5}$ is the second transient Earth Trojan known. To study its orbit, we used archival data from 2012 to 2019 and observed the object in 2021 from three groundbased observatories. Our study of its orbital stability shows that $2020 \mathrm{XL}_{5}$ will remain in $L_{4}$ for at least 4000 years. With a photometric analysis we estimate its absolute magnitude to be $H_{r}=18.58_{-0.15}^{+0.16}$, and color indices suggestive of a C-complex taxonomy. Assuming an albedo of $0.06 \pm 0.03$, we obtain a diameter of $1.18 \pm 0.08 \mathrm{~km}$, larger than the first known Earth Trojan asteroid.

\footnotetext{
${ }^{1}$ Departamento de Fisica, Ingeniería de Sistemas y Teoría de la Señal, Universidad de Alicante, Carr. de San Vicente del Raspeig, s/n, 03690 San Vicente del Raspeig, Alicante, Spain. ${ }^{2}$ Institut de Ciències del Cosmos (ICCUB), Universitat de Barcelona (IEEC-UB), Carrer de Martí i Franquès, 1,08028 Barcelona, Spain. ${ }^{3}$ ESA NEO Coordination Centre, Largo Galileo Galilei, 1, 00044 Frascati, Italy. ${ }^{4}$ Arecibo Observatory, University of Central Florida, HC3 Box 53995, Arecibo, PR 00612, USA. ${ }^{5}$ Instituto de Astrofísica de Andalucía, CSIC, Apartado 3004, 18080 Granada, Spain. ${ }^{6}$ Instituto de Física Aplicada a las Ciencias y las Tecnologías, Universidad de Alicante, San Vicente del Raspeig, 03080 Alicante, Spain. ${ }^{7}$ Observatório Nacional / MCTIC, R. Gen. José Cristino, 77, Rio de Janeiro 20921-400, Brazil. ${ }^{8}$ Astronomical Observatory Institute, Faculty of Physics, A. Mickiewicz University, Słoneczna 36, 60-286 Poznań, Poland. ${ }^{9}$ Solenix Deutschland GmbH, Spreestraße 3, 64295 Darmstadt, Germany. ${ }^{10}$ The University of Arizona, Lunar and Planetary Laboratory, 1629 E University Blvd, Tucson, AZ 85721, USA. ${ }^{11}$ Institute for Astronomy, University of Hawaii, 2680 Woodlawn Dr, Honolulu, HI 96822, USA.

${ }^{12}$ Department of Physics and Astronomy, The University of Western Ontario, 1151 Richmond St, London, ON N6A 3K7, Canada. ${ }^{13}$ Laboratório Nacional de Astrofísica LNA/MCTIC, R. dos Estados Unidos, 154, Itajubá 37504-364, Brazil. ${ }^{14}$ Cerro Tololo Inter-American Observatory/NSF's NOIRLab, Casilla 603, La

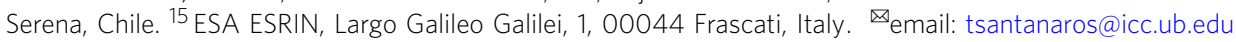




\section{T} he classical work of J. L. Lagrange published in 1772 on the three-body problem had to wait until 1906 to find an empirical verification with the discovery of asteroid (588) Achilles. This asteroid is orbiting around a theoretical point located $60^{\circ}$ ahead of Jupiter along its orbit. After discovering (588) Achilles, many other objects were found orbiting around nearly the same point or its mirroring position $60^{\circ}$ behind Jupiter. Both points are the so-called triangular Lagrange points and are commonly known as $L_{4}$ (for the former) and $L_{5}$ (for the latter) ${ }^{1}$. Asteroids orbiting around these points of a planet-Sun system are known as Trojan asteroids.

Although Trojan asteroids have been known for decades in other Solar System planets ${ }^{2}$ such as Venus ${ }^{3}$, Mars ${ }^{4}$, Jupiter ${ }^{5}$, Uranus $^{6}$, and Neptune ${ }^{7}$, it wasn't until 2011 that asteroid 2010 $\mathrm{TK}_{7}$ was found to be the first (and hitherto unique) Earth Trojan (ET) asteroid ${ }^{8}$. ET asteroids have been broadly debated and proved to be feasible from the point of view of celestial mechanics. In particular, their possible existence has been shown using theoretical studies by means of numerical simulations ${ }^{9,10}$. Observational strategies have been defined trying to detect new ETs but all the dedicated surveys performed so far have failed to discover any new member of this population ${ }^{11-14}$, including an in situ survey ${ }^{15}$ performed by the OSIRIS-REx spacecraft within the $L_{4}$ region and observations ${ }^{16}$ of $L_{5}$ by the Hayabusa 2 spacecraft on its way to asteroid (162173) Ryugu. Despite failing in the detection of new ET asteroids, some of these surveys provided population constraints regarding their number and their size $\mathrm{s}^{12-15}$.

The reason behind this low discovery success rate is related to the unfavorable viewing geometry of an object orbiting EarthSun's $L_{4}$ or $L_{5}$ points as seen from our planet ${ }^{17}$. In short, these objects are often observable very close to the Sun (i.e., at low Solar elongations) and under large phase angles (the Sun-objectobserver angle), meaning that a significant fraction of the object is shadowed as seen from the Earth, which in turn implies the object being faint. Under such geometries, observations must be acquired at high airmass, where seeing is typically worse, which, together with higher background from zodiacal light, further increases the difficulty of these searches. For both ETs known to date, opportunities for better observing geometries at larger solar elongations might exist thanks to their higher eccentricity values.

Asteroid $2020 \mathrm{XL}_{5}$ was discovered by the Pan-STARRS1 survey on 2020 December 12. Shortly after the discovery, follow-up observations were gathered from different stations, allowing for an initial orbit determination. The orbit behavior suggested that $2020 \mathrm{XL}_{5}$ could have been a candidate to become the second known ET, but the orbit uncertainty due to the short arc covered with observations was still too large to confirm a current Trojan engagement with Earth ${ }^{18}$.

Here we present the results of our study based on our new observations of $2020 \mathrm{XL}_{5}$ which confirms that $2020 \mathrm{XL}_{5}$ will be an ET for at least 4000 years.

\section{Results}

Orbit stability. The nominal orbit of $2020 \mathrm{XL}_{5}$ has been computed at the epoch $\mathrm{MJD}=58444.1$ using the European Space Agency's (ESA) AstOD orbit determination software ${ }^{19,20}$, based on the methods described in the literature ${ }^{21}$, taking as input the full observations dataset described in the observation sections of Methods. The resulting Keplerian orbital elements and their uncertainties are presented in Table 1 . The dynamical model used in the orbit determination and propagation takes into account the $N$-body gravitational attraction of the Sun, eight planets,

\section{Table 1 Orbital elements.}

\begin{tabular}{llll} 
Element & Value & $\mathbf{1} \boldsymbol{\sigma}$ uncertainty & Unit \\
\hline$a$ & 1.00070559767 & $5.61 \times 10^{-9}$ & $\mathrm{au}$ \\
$e$ & 0.387220870 & $1.56 \times 10^{-7}$ & \\
$i$ & 13.8458718 & $1.58 \times 10^{-5}$ & $\mathrm{deg}$ \\
$\Omega$ & 153.6128174 & $5.03 \times 10^{-5}$ & $\mathrm{deg}$ \\
$\omega$ & 87.9797957 & $4.25 \times 10^{-5}$ & $\mathrm{deg}$ \\
$M$ & 258.3840814 & $2.25 \times 10^{-5}$ & $\mathrm{deg}$ \\
\hline $\begin{array}{l}\text { Keplerian orbital elements of 2020 XL5 and their uncertainties, at the epoch MJD }=58444.1, \\
\text { computed with the European Space Agency's (ESA) AstOD orbit determination software }{ }^{19,20} \\
\text { based on the methods described in the literature } \\
\text { described in the observation sections of Methods. }\end{array}$ \\
\hline
\end{tabular}

the Earth's Moon, and the parameterized post-Newtonian relativistic contribution and the oblateness of Sun and Earth. The software makes use of the JPL Planetary and Lunar Ephemerides $\mathrm{DE} 431^{22}$.

In order to investigate the stability of the object in the $L_{4}$ Lagrange point of the Earth, we performed a set of numerical simulations by integrating the nominal orbit, together with 800 clone orbits sampling its uncertainty, over a time span of $\sim 29,000$ years. The integration of the orbits has been performed using the ESA AstOD orbit determination software. The 800 clone orbits have been computed by sampling the orbit covariance matrix. The Trojan-like behavior of an object is seen in a reference frame co-rotating with the Earth's orbital motion ${ }^{23}$. The key parameter to quantify the state is the relative mean longitude $\lambda_{r}$ is defined as the difference between the mean longitude of the asteroid $\lambda_{a}$ and the mean longitude of the Earth $\lambda_{E}$ : when the resonant angle $\lambda_{r}$ librates around $60^{\circ}$, i.e., $0^{\circ}<\lambda_{r}<180^{\circ}$ the asteroid is called an $L_{4}$ Trojan, when the resonant angle $\lambda_{r}$ librates around $300^{\circ}$, i.e., $180^{\circ}<\lambda_{r}<360^{\circ}$ the asteroid is called an $L_{5}$ Trojan, when $\lambda_{r}$ circulates then the asteroid leaves the Trojan-like orbit ${ }^{24}$. Nevertheless, Trojans can have a displacement of a maximum of $\omega=25^{\circ} 11^{\prime}$ from the typical equilateral location for eccentric orbits $^{25}$. We found that in the case of $2020 \mathrm{XL}_{5}$ the mean longitude $\left(\lambda_{r}\right)$ evolution of the nominal orbit in Table 1 shows a transient ET behavior for this object (see Fig. 1). We define $t_{0}=0$ as the mean epoch of the observations arc (corresponding to November 2018). The plot shows that before the time $t_{1} \simeq-500$ years, $\lambda_{r}$ circulates, and therefore the asteroid could not be considered to be in a Trojan-like orbit. Starting from $t_{1}, 2020 \mathrm{XL}_{5}$ is an $L_{4}$ ET librating around $\lambda_{r} \sim 75^{\circ}$, being stably located at the Lagrangian point for a time interval of about 4500 years, until the time $t_{2} \simeq 4000$.

The observed stability time interval of the clone orbits appears to be consistent with that of the nominal orbit, as shown in Fig. 1, where a different color for each clone orbit has been used, with the green line representing the nominal orbit. Some clone orbits escape from stability before the nominal one, as shown in the zoomed Fig. 2.

To better visualize the results we generated a stack plot classifying the behavior of the nominal and clone orbits in three different states: librating around $L_{4}$, librating around $L_{5}$, and circulating (Fig. 1). The stack plot covers the entire time span of the simulations, with the time $t_{0}=0$ set as the mean epoch of the observation arch. The plot shows that some orbits begin to show instability after time $t \sim 3500$, and by time $t=5000$ less than $40 \%$ of the orbits are still in $L_{4}$. Integrating backward, all the orbits become unstable by time $t \sim-500$, and less than $5 \%$ of orbits are located in $L_{4}$ by $t \sim-2000$ and earlier. Moreover, the plot shows that less than $10 \%$ of the orbits are librating around $L_{5}$ both before $t \sim-1000$ and during the interval between $t \sim 6000$ and $t \sim 8000$. 


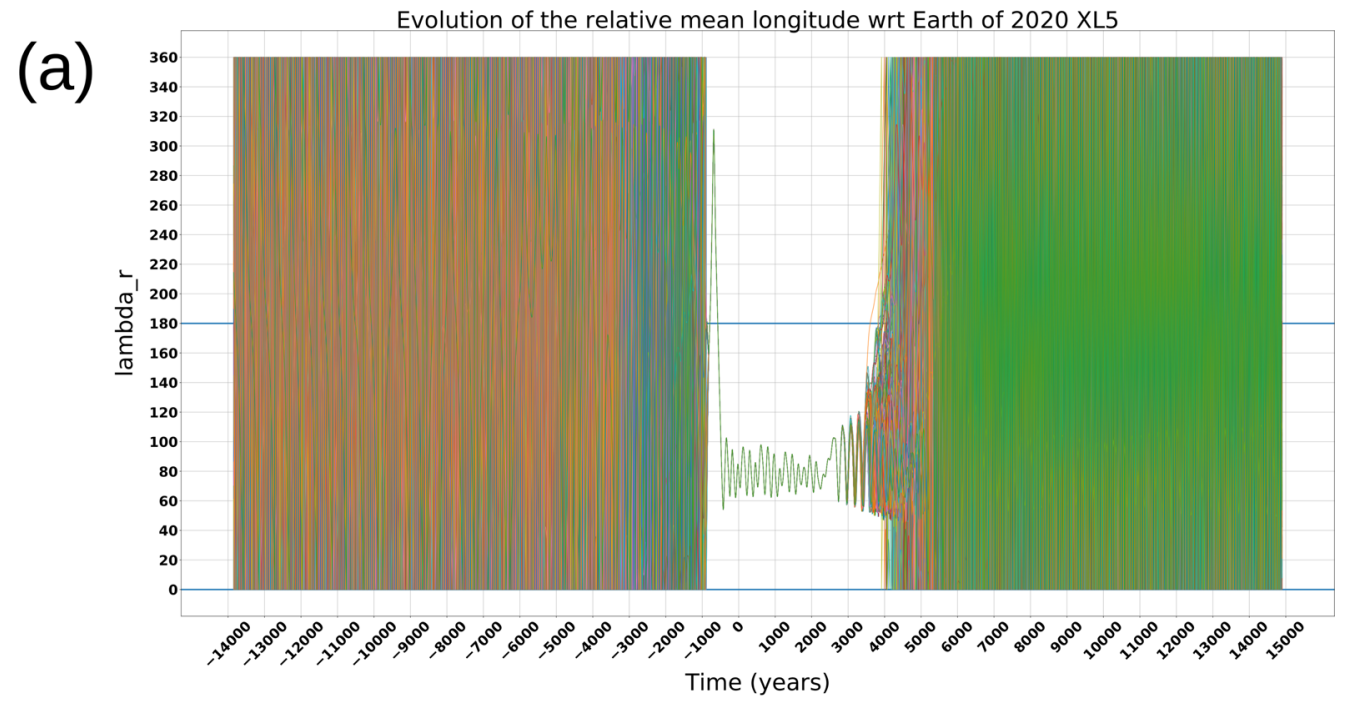

(b)
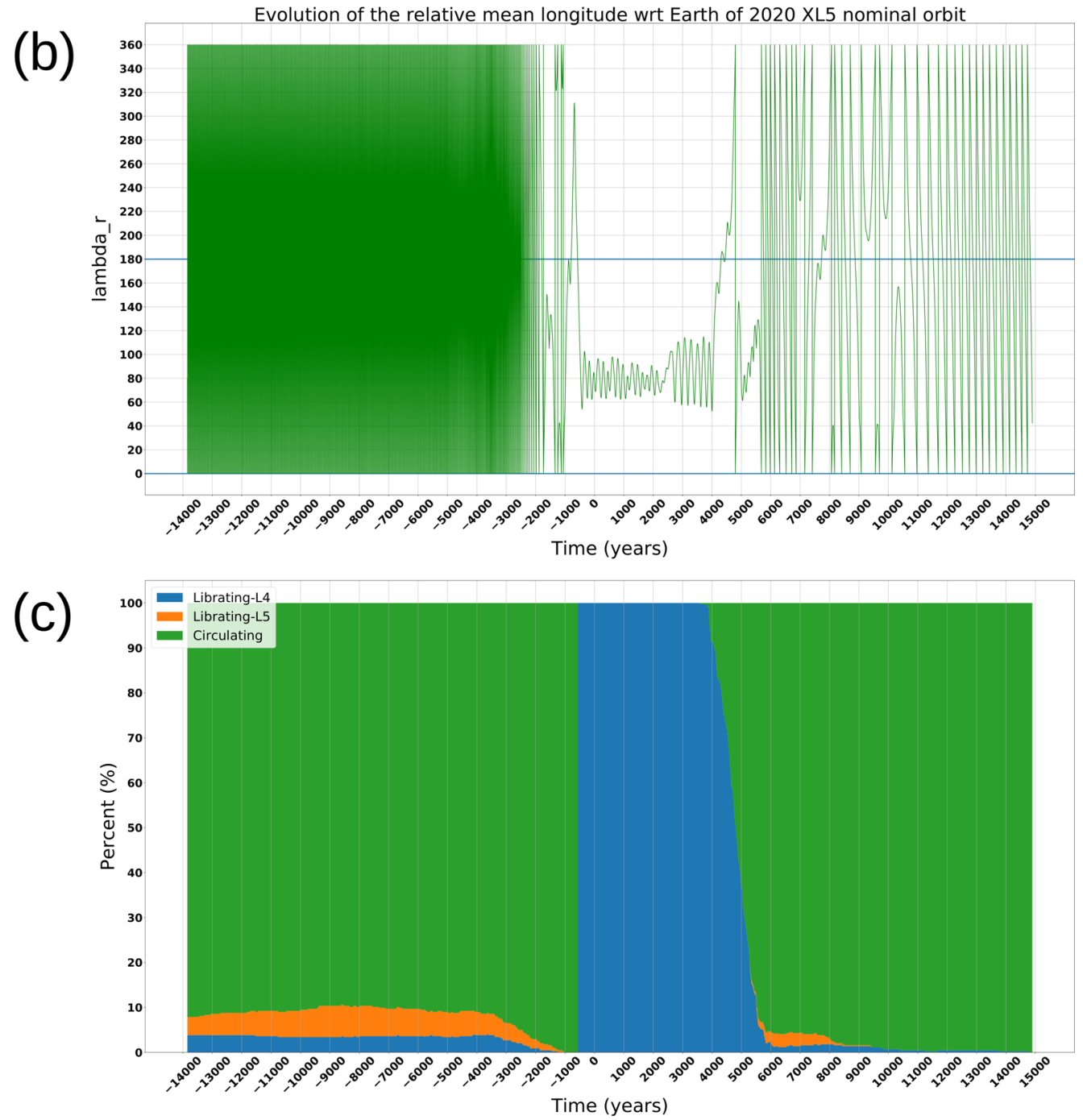

Fig. 1 Mean longitude evolution analysis. a The relative mean longitude $\left(\lambda_{r}\right)$ evolution of the $2020 \mathrm{XL}_{5}$ nominal orbit as in Table 1 and clones orbit over 29,000 years, where each clone orbit is represented by a different color, while the green line represents the nominal orbit. $\mathbf{b}$ Evolution of $\lambda_{r}$ for the nominal orbit of $2020 \mathrm{XL}_{5}$ over 29,000 years. c Stack plot representing the behavior of the nominal orbit and the 800 clone orbits. In the plot the time $t_{0}=0$ is the mean epoch of the orbits. Source data are provided as a Source Data file. 


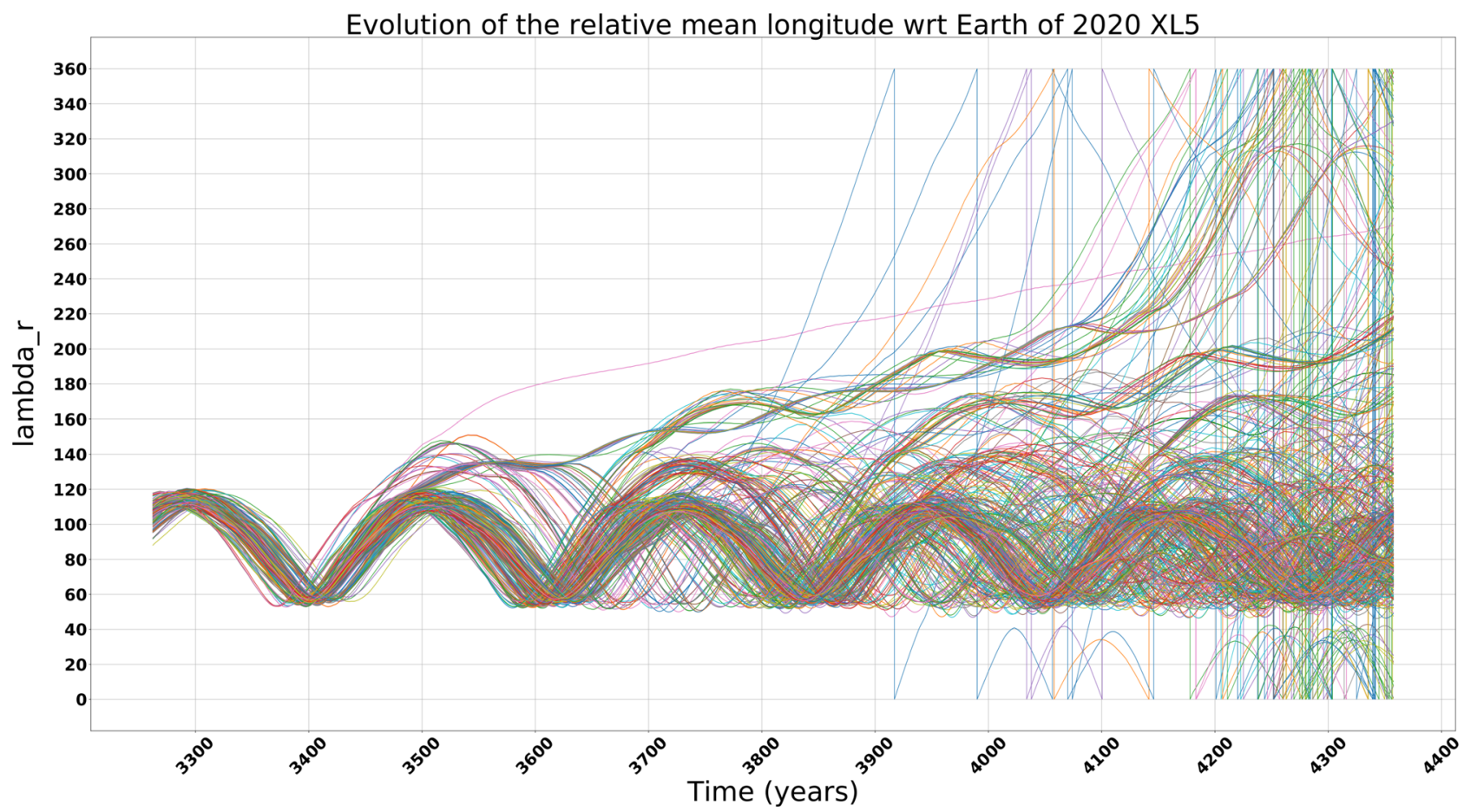

Fig. 2 Beginning of the non-deterministic regime. The relative mean longitude $\left(\lambda_{r}\right)$ evolution of the $2020 X L_{5}$ nominal orbit as in Table 1 and clones orbit from year 3300 until year $\sim 4300$, where each clone orbit is represented by a different color. Some clone orbits escape from the stability before the nominal one. Source data are provided as a Source Data file.

These transitions between libration points are a well-known behavior for objects in co-orbital motion, especially for those in orbits with large enough eccentricity and/or high enough inclination ${ }^{26}$.

Physical characterization. In addition to the orbital analysis discussed above, we also investigated the physical characteristics of $2020 \mathrm{XL}_{5}$, by photometrically analyzing the new imaging data gathered during the 2020-2021 apparition and the precovery datasets. The SNR of the target was not sufficient to perform time-series photometry, since the object was only marginally detectable on single images, and could only be measured after stacking multiple frames. Moreover, since the observations were obtained close to dawn and with the telescope pointing very low over the horizon due to the object's proximity to the Sun, the background signal increased very fast from frame to frame, and only the first 4 images for each band were useful for photometric purposes. Nevertheless, we obtained V-band photometry on 2021 February 22, Sloan $\mathrm{g}^{\prime}, \mathrm{r}^{\prime}$, and $\mathrm{i}^{\prime}$ photometry on 2021 March 9, and Sloan $\mathrm{r}^{\prime}$ photometry on 2021 March 13 and 16. Using these measurements, we investigated the taxonomy of the object; it is compatible with the C-complex, as shown in Fig. 3, although the low SNR of the measurements results in a large uncertainty for all the determined colors. The computed $\mathrm{a}^{*}$ color is $a^{*}=-0.9 \pm 0.6$ for the first night and $a^{*}=-0.4 \pm 1.0$ for the second night, which are compatible with C-complex objects. The S-complex objects typically have $a^{*}>0$ and on average $a^{*} \sim 0.15$ and the C-complex objects $a^{*}<0$ with average value of $a^{*} \sim-0.1^{27}$. In addition, deviations due to lightcurve amplitude effects could not be corrected, since the spin state of the object is still unknown. In light of the significant uncertainty of the photometric measurements, and the impossibility to compensate for rotational effects, this taxonomic classification is to be seen as provisional, and needs to be confirmed by further observations, ideally when the object presents a more favorable viewing geometry. We further

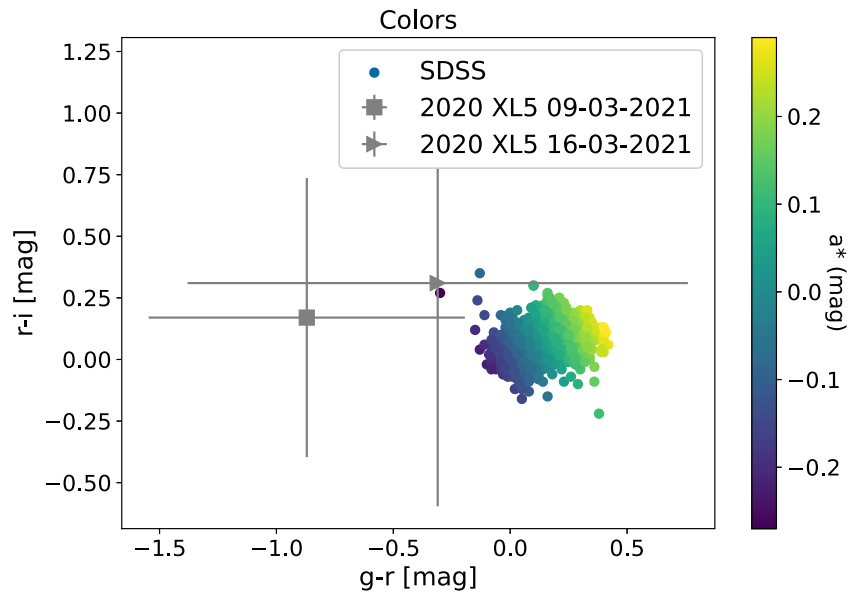

Fig. 3 Color indices. The $g-r$ and $r-i$ colors indices of $2020 \mathrm{XL}_{5}$ from the two observing nights, the color scale is according to the SDSS $a^{\star}$ parameter. Gray lines represent one sigma color uncertainty. The 2043 color points represent 1308 asteroids from all taxonomic types present in the SDSS MOC DR3 catalog. The C-complex objects typically have $a^{\star}<0$ with an average value of -0.1 and the S-complex object $a^{\star}>0$ with an average value of $0.15^{27} .2020 \mathrm{XL}_{5}$ is located in the $\mathrm{C}$-complex area. Source data are provided as a Source Data file.

investigated the photometric behavior of the object by analyzing the dependence of its brightness on the phase angle at the different nights of observations, to obtain the object's phase curve (see Methods subsection Phase curve). After calibrating the data we obtained $H_{r}=18.58_{-0.15}^{+0.16}$ for a C-type asteroid ${ }^{28}$ with $G_{1_{r}}^{*}=$ 0.83 and $G_{2_{r}}^{*}=0.02$. Thus, assuming a C-complex class, and therefore an albedo of $0.06 \pm 0.03^{29}$, we estimate the diameter of 


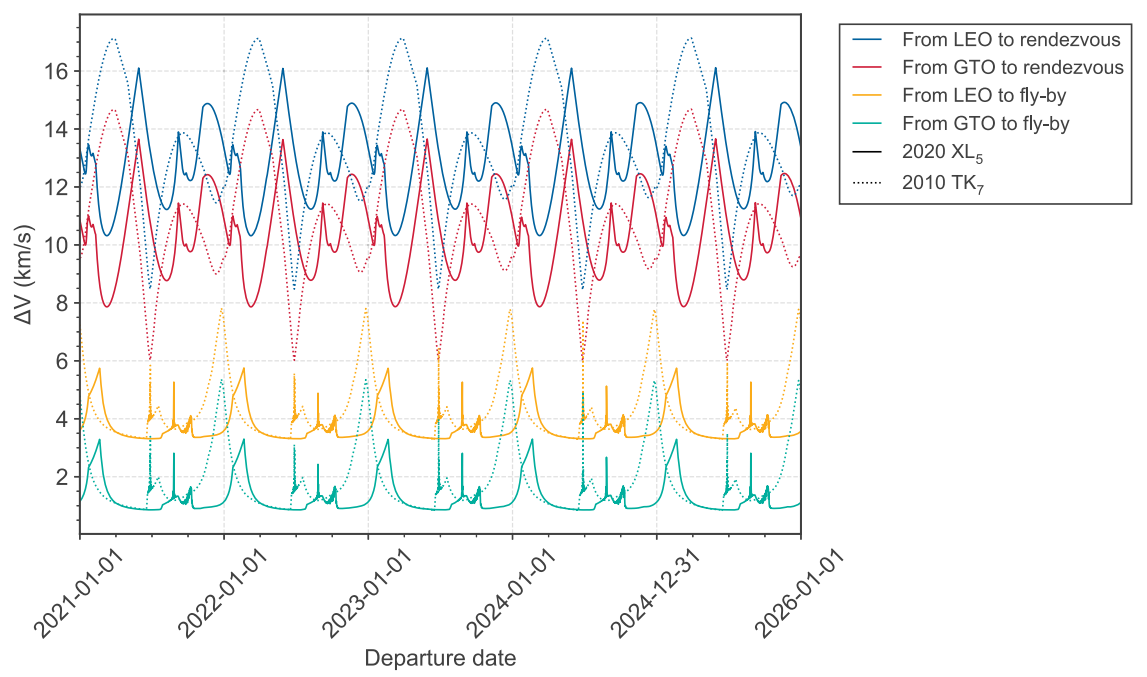

Fig. 4 Delta-V of trajectories. Minimum delta-v trajectories to $2020 \mathrm{XL}_{5}$ (solid line) and $2010 \mathrm{TK}_{7}$ (dotted line) for the next 5 years. Four different scenarios are considered: departure from LEO and/or GTO orbit, and a rendezvous and/or fly-by mission to the asteroid. Source data are provided as a Source Data file.

$2020 \mathrm{XL}_{5}$ as $1.18_{-0.08}^{+0.08} \mathrm{~km}$. The uncertainty interval corresponds to the 16th and the 84 th percentile, encompassing the $68 \%$ of the underling distribution of possible values. The inferred diameter for $2020 \mathrm{XL}_{5}$ is larger than the value known for the first ET asteroid, $2010 \mathrm{TK}_{7}$, which was estimated ${ }^{8}$ to have a diameter of $\sim 0.3 \mathrm{~km}$.

Delta-v budget. ETs, among the Earth co-orbital objects, are considered to be potential candidates for rendezvous and even sample return missions, due to the low-energy requirements expected, as shown in previous works ${ }^{30}$ with a theoretical population of ETs. Therefore, we decided to investigate the required delta-v budget for both a rendezvous and a fly-by mission to the two ETs known, $2020 \mathrm{XL}_{5}$ and $2010 \mathrm{TK}_{7}$, in order to decide if they would be good candidates for a mission. The pykep tool $^{31}$ used for the analysis is described in the Methods subsection Delta-v budget.

Figure 4 shows, for both objects, the minimum total delta-v required for each departure date and each of the considered scenarios, i.e., a launch from Low Earth orbit (LEO) or Geostationary transfer orbit (GTO), and a space mission to a rendezvous or a fly-by with the asteroid.

First, for a rendezvous mission to $2020 \mathrm{XL}_{5}$, the absolute minimum total delta- $\mathrm{v}$ is estimated to be between 7.9 and $10.3 \mathrm{~km} / \mathrm{s}$, depending on the launch conditions. Launching from LEO directly to escape is very expensive, and thus, the resulting required delta-v budget is not feasible. Getting to the GTO orbit via a shared launch significantly reduces the delta- $v$ down to a value lower than $8 \mathrm{~km} / \mathrm{s}$, but it is still too high to be considered an ideal target for a rendezvous mission.

When comparing the results for $2020 \mathrm{XL}_{5}$ to the other known ET, we can see that the latter presents slightly lower values for the absolute minimum total delta-v, which is estimated to be between 6 and $8.5 \mathrm{~km} / \mathrm{s}$, also far from the expected very low-energy requirements for rendezvous to theoretical $\mathrm{ETs}^{30}$. The main reason for such large velocities is the relatively high inclination of both $2020 \mathrm{XL}_{5}$ and $2010 \mathrm{TK}_{7}$, resulting in an additional planechange maneuver that is extremely costly. In a previous largescale statistical survey of delta-v to Earth co-orbital asteroids, similar conclusions were derived regarding the importance of inclination when considering a low delta-v budget to rendezvous asteroids with Earth-like orbits ${ }^{30}$. It might be preferable to consider another Earth co-orbital object closer to Earth's orbital plane, or instead of a rendezvous mission, consider a fly-by mission to the asteroid. Therefore, we conclude that neither of the known ETs are good candidates for a space mission.

Figure 4 also shows the total delta-v required to perform a flyby, which is significantly lower than for a rendezvous with the asteroid, since there is no need to match the asteroid's orbit and hence no additional maneuver is performed. In addition, similar absolute minimum total delta-v values are obtained for both ETs, between 0.9 and $3.3 \mathrm{~km} / \mathrm{s}$ depending on the launch conditions, making both of them potential fly-by targets. It is however important to highlight that $2020 \mathrm{XL}_{5}$ presents a flatter minimum total delta-v with respect to the departure date. Therefore, $2020 \mathrm{XL}_{5}$ might be a better candidate for a fly-by mission to an ET since it provides more flexibility to choose a suitable launch date.

\section{Discussion}

Several observational surveys have been devoted to discover ETs near the L4 and L5 points ${ }^{12-16}$. Despite these efforts, only two objects have been discovered so far: $2010 \mathrm{TK}_{7}$ and $2020 \mathrm{XL}_{5}$. However, both asteroids are transient ETs, meaning that their stability around L4 has been shown to be in the scale of thousands of years, far from the stability time-scale of a theoretical primordial ET population, which are thought to be remnants from the Earth's formation period ${ }^{32}$. Although no primordial ETs has been found yet, some constraints have been provided on their population. The most recent and restrictive values on their magnitude limit for $\mathrm{L} 4^{14}$ are $N_{E T}<1$ for $H=13.93, N_{E T}<10$ for $H=16$, and $N_{E T}<938$ for $H=22$, while for $\mathrm{L} 5^{13}$ are $N_{E T}<1$ for $H<15.5, N_{E T}=60-85$ for $H<19.7$, and $N_{E T}=97$ for $H=20.4$.

The discovery of a second ET asteroid may enhance our knowledge of the dynamics of this elusive population. By comparing the orbital nature of the two ETs known so far, we can better understand the mechanisms that allow for their transient stability. For instance, the librating point of both asteroids is displaced from the expected $60^{\circ}$ due to their inclined and eccentric orbits. This might suggest that captured ET asteroids may likely be found in orbits displaced from the libration points. 
Regarding the physical characterization of $2020 \mathrm{XL}_{5}$, the improvement of its orbit and therefore its ephemeris to the arcsecond level provided by this work is opening new interesting observational possibilities. In particular, it is now possible to plan observations with instruments having small fields of view. For instance, gathering new photometric data of the object during its yearly favorable observing window from November to December, will help to reduce the uncertainty on the color indices and therefore enhance its taxonomic classification. More specific studies resulting from infrared or spectroscopic observations will also enhance our knowledge of this dynamically exotic object. The latter will provide better constraints on its size estimation and composition. If follow-up observations confirm its C-complex nature, one reasonable explanation of this body's orbit would be a transient capture from the main belt, after being ejected from the main belt by any of the $2: 1$ and 5:2 resonance complex with Jupiter ${ }^{33}$. Nonetheless, it has been shown ${ }^{34}$ that the Hungaria region could be a possible source of co-orbital bodies in the inner Solar System, with libration periods around $\sim 10 \mathrm{kyr}$, in agreement with our results for $2020 \mathrm{XL}_{5}$. On top of that, it has been found ${ }^{35}$ that the Hungaria region is mostly dominated by $\mathrm{C}$ and S-types, especially at small sizes, which is also consistent with our taxonomic classification of $2020 \mathrm{XL}_{5}$.

Future surveys of the L4 and L5 regions will allow to derive tighter constraints on the ET populations and, maybe, discover their primordial bodies. Their study could help enhance the Solar System evolution models and therefore can be very valuable to understand its formation. Discovering ETs having lower orbital inclination and eccentricity might have another important implication: unlike $2020 \mathrm{XL}_{5}$, objects librating near to the Lagrangian points with low inclinations could be reached from the Earth with a very low delta-v budget ${ }^{30}$. Therefore these objects may become ideal targets for space missions and, in the more distant future, to settle human bases or install scientific hardware that would benefit from their peculiar location.

\section{Methods}

New observations. The astrometric dataset of $2020 \mathrm{XL}_{5}$, including information about the telescopes and the observing conditions, is presented in Table 2 . In the following section, we describe the observations performed in our follow-up observation campaign for each of the telescopes used.

In order to extend the observed arc of $2020 \mathrm{XL}_{5}$ into 2021, we gathered optical images of $2020 \mathrm{XL}_{5}$ with two $4 \mathrm{~m}$ class telescopes (the Southern Astrophysical Research telescope and the Lowell Discovery Telescope) and a $1.0 \mathrm{~m}$ telescope (ESA's Optical Ground Station) from 2021 February 9 to 2021 March 16, covering an additional orbital arc of 35 days.

The target's viewing geometry and observational circumstances were extremely challenging during the time of all these observations: the target was very close to the Sun (low elongation), and also overlapping with the galactic plane earlier in the observation period.

We designed our observing strategy for the purely astrometric datasets prioritizing the goal of achieving the minimum signal-to-noise ratio (SNR) necessary in order to make the object measurable from an astrometric point of view. We, therefore, used broad-band filters in all our observations and pointed to the object as soon as it reached the minimum elevation allowed by the telescopes' specifications. All in all, we had a few minutes per night to observe the object until the background quickly started saturating due to twilight.

Additionally, the length of the exposure times was limited by the apparent motion of $2020 \mathrm{XL}_{5}$. Therefore to achieve the best possible astrometric accuracy, while still keeping the ability to track non-linearly on the motion of the object, and reject bright overlapping stars, we decided to limit our single exposures to at most 20 s. Astrometric measurements were then extracted from stacked images for each night, whereas a quality test for the background levels was performed to select the frames qualifying for the photometric analysis.

Optical ground station (OGS). ESA's Optical Ground Station (OGS) $1.0 \mathrm{~m}$ telescope (MPC code J04) in Tenerife, Canary Island, Spain, is regularly used by ESA's Planetary Defence Office to obtain astrometric observations of NEOs. In the context of such routine monitoring and follow-up activities, our team obtained and reported observations of the target on 2020 December 13, providing observational confirmation of the object's existence just $14 \mathrm{~h}$ after the initial discovery. Other stations reported additional data over the following nights, but the observational

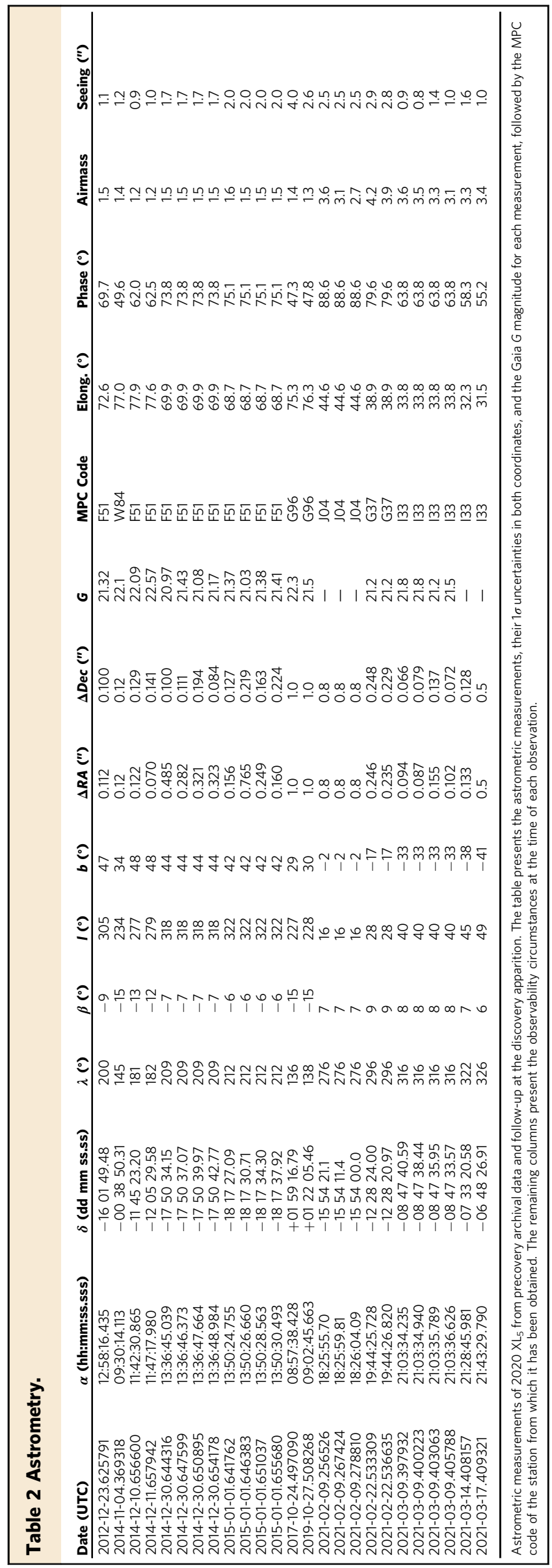


coverage available at the Minor Planet Center ended in early 2021 January. By the end of the month, the object's trajectory brought it towards the skyplane location of the galactic center, and observations became consequently more challenging. We nevertheless decided to attempt further observations in early February with the OGS telescope. The field containing the object was exposed on three consecutive mornings, from 2021 February 9 to 11 . On the first night, we could detect a possible faint candidate on a small subset of frames not significantly affected by background stars. On the subsequent nights, the stellar confusion of the field was too significant to achieve any detection. All the OGS observations were obtained with the ESA Space Debris Camera 2 (SDC2), equipped with a $4 \mathrm{k}$ imager used in $2 \times 2$ binning mode and sidereal tracking. This instrument configuration results in a $47.5^{\prime}$ FoV, with $1.39^{\prime \prime}$ binned pixels, optimally sampling the $\sim 2.5^{\prime \prime}$ to $3^{\prime \prime}$ FWHM of the system's PSF for astrometric purposes.

Lowell discovery telescope (LDT). Observations at the $4.3 \mathrm{~m}$ Lowell Discovery Telescope (G37) were obtained on 2021 February 22 during astronomical twilight when $2020 \mathrm{XL}_{5}$ was at an airmass between 4.2 and 3.8. We used the Large Monolithic Imager (LMI) ${ }^{36,37}$. LMI is a e2v CCD231-SN-10382-14-0 of $6144 \times 616015 \mu \mathrm{m}$ pixels. On the LDT, LMI provides a field of view of $12.3^{\prime} \times 12.3^{\prime}$ with a pixel scale of $0.12^{\prime \prime} /$ pixel when operated unbinned. For the $2020 \mathrm{XL}_{5}$ observation we used a binning mode of $5 \times 5$ providing a pixel scale of $0.60^{\prime \prime} /$ pixel. We obtained 13 individual exposures of $20 \mathrm{~s}$ each. The telescope was tracking at a non-sidereal rate matching the motion of the asteroid of $3.44^{\prime \prime} /$ pixel providing star trails of $1.15^{\prime \prime}$ that are much smaller than the $\sim 3^{\prime \prime}$ seeing at such high airmass. The observations were performed in the VR filter (bandpass from $0.480 \pm 0.005$ to $0.721 \pm 0.005 \mu \mathrm{m})$. The resulting stacked image is shown in Fig. 5 .

Southern astrophysical research (SOAR). We used the $4.1 \mathrm{~m}$ Southern Astrophysical Research Telescope (I33) on the nights of 2021 March 9 (NOIRLab program 2018B-927, P.I., S. Zepf, Michigan State, University), 13 (NOIRLab Astronomical Event Observatory Network (AEON) 2021A queue), and 16 (Brazil DDT night, PI: L. Fraga). We used the Goodman optical imager which provides a field of view of $7.2^{\prime}$ and a pixel scale of $0.15^{\prime \prime} /$ pix. Observations were very challenging due to the extremely low solar elongation of the object at that time (between $32^{\circ}$ and $34^{\circ}$ away from the Sun). As a result, the object was observable only during a few minutes before dawn and very close to the horizon ( $15^{\circ}$ elevation), with an airmass between 3.7 and 3.3. We used the Goodman HTS imaging camera equipped with SDSS filters and $3 \times 3$ binning mode in order to reduce the readout time. We tracked non-sidereally and used short exposures $(20 \mathrm{~s})$ for each of the $\mathrm{g}^{\prime}, \mathrm{r}^{\prime}$, and $\mathrm{i}^{\prime}$ filters. The background was quickly saturating and only the first four frames of each band were useful for photometric analyses.

Additional observational attempts. We also attempted observations of $2020 \mathrm{XL}_{5}$ using the $0.8 \mathrm{~m}$ Joan Oró (TJO) telescope (MPC code C65) and the $2.2 \mathrm{~m}$ Calar Alto (CAHA) telescope (MPC code 493) on 2021 February 11 and 2021 March 2 respectively. However, on both attempts, we could not confidently detect the object, even after stacking all the frames, and therefore we are not including these data in the analyses presented in this work.

Precovery observations. The new observational data gathered by our team and discussed above, combined with the astrometry available at the Minor Planet Center, cover the entire observable arc from the time of the earliest submitted precovery observation (2020 November 26 , by code G96), to the disappearance of the object into solar conjunction (elongation $<30^{\circ}$, at the end of $2021 \mathrm{March}$ ), which marked the end of the discovery apparition. Overall, the arc covers almost 111 days, sufficient for a rough analysis of the stability of the object, but not ideal for a long-term study of the behavior of the object ${ }^{18}$.

In order to increase the observed arc without the need to wait for an additional future apparition, we performed a thorough search for precovery detections in archival data. We began with the archive of the Catalina Sky Survey: the search revealed a promising field exposed with the $1.5 \mathrm{~m} \mathrm{Mt}$. Lemmon telescope (G96) on 2019 October 27, at a time when the object had an ephemeris uncertainty of just $\pm 5^{\prime \prime}$ ( $1 \sigma$ confidence) based on the data from the 2020-2021 apparition. A careful analysis of the area of the images corresponding to the prediction revealed a possible faint candidate with a signal-to-noise ratio (SNR) of $\sim 3$.

Despite not being a convincing detection, we temporarily assumed its correctness and used it to extend our search further back in time. Another promising field was located, exposed by the same telescope on 2017 October 24 . The uncertainty of the ephemeris at that time, assuming the correctness of the 2019 detection, was only $\pm 2.3^{\prime \prime}$. No source was visible in the single images, but another very faint source with SNR $\sim 3$ appeared in the stack of all four frames of that night. These two tentative detections, individually not sufficiently strong to claim a certain identification, when taken together provide reasonable evidence that they could indeed be real. Their orbit improvement potential was so great that they now made it possible to determine the object's ephemeris to better than $\pm 5^{\prime \prime}$ as far back as 2012, allowing for many additional precovery opportunities at multiple apparitions.

We, therefore, extended our search to two additional archives: the online repository of all images obtained by the wide-field DECam instrument with the $4.1 \mathrm{~m}$ Víctor M. Blanco Telescope on Cerro Tololo, Chile, and the archive of the
$1.8 \mathrm{~m}$ Pan-STARRS survey. The DECam archive provided a solid detection compatible with the tentative G96 precoveries on 2014 November 4, while PanSTARRS thoroughly confirmed the chain of precovery observations providing eleven additional measurements covering a time span from 2012 to 2015 (see Fig. 5).

Orbit determination. Clone orbits used in the numerical simulations were generated applying the Cholesky method for multivariate normal distributions ${ }^{38}$. The clone orbits are generated using Python $3.6^{39}$, starting from the nominal orbit and its covariance matrix computed with the AstOD software. Alternatively, freely available software such as $\mathrm{OrbFit}^{40}$ can be used for the analysis.

The Cholesky method consists of the factorization of a Hermitian, positivedefinite matrix, as the product of a lower triangular matrix and its transpose conjugate. In our case, the covariance matrix $C$ is thus decomposed as $C=L L^{T}$, where $L$ is a lower triangular matrix. The Keplerian orbital elements $\mathbf{e}_{\mathbf{i}}$ of the $i$-th clone orbit are then defined as follow:

$$
\mathbf{e}_{i}=\mathbf{e}_{0}+L \mathbf{r}_{\mathbf{i}}
$$

where $\mathbf{e}_{\mathbf{0}}$ is the vector of the orbital elements of the nominal orbit, and $\mathbf{r}_{\mathbf{i}}$ is a 6-dim random vector, whose components are generated following a normal distribution with mean 0 and variance $1(\mathcal{N}(0,1))$.

Since the uncertainties in the nominal orbital elements are very small differences between the initial conditions of the clone orbits and those of the nominal orbit are small as well.

Starting from these initial conditions, we have integrated the 800 clone orbits along a time span of $\sim 29,000$ years. Considering as initial time $t_{0}=0$ the mean epoch of the observations, the forward propagation has been executed along 15,000 years, where this limit in time is defined by the JPL Planetary and Lunar Ephemerides DE43122 whilst the backward propagation has been executed for 14,000 years. As a second step, we studied the evolution of the relative mean longitude $\lambda_{r}$ of $2020 \mathrm{XL}_{5}$ with respect to the Earth. The relative mean longitude $\lambda_{r}$ is defined as the difference between the mean longitude of the asteroid $\lambda_{a}$ and the mean longitude of the Earth $\lambda_{E}$ : when the resonant angle $\lambda_{r}$ librates around $60^{\circ}$, i.e., $0^{\circ}<\lambda_{r}<180^{\circ}$ the asteroid is called an $L_{4}$ Trojan, when the resonant angle $\lambda_{r}$ librates around $300^{\circ}$, i.e., $180^{\circ}<\lambda_{r}<360^{\circ}$ the asteroid is called an $L_{5}$ Trojan, when $\lambda_{r}$ circulates then the asteroid leaves the Trojan-like orbit ${ }^{24}$

We have reproduced the same calculations using the public software OrbFit ${ }^{40}$, obtaining consistent results for the deterministic part of the orbital evolution.

Validation of the orbital study method. In order to validate the methods adopted during the analysis of the orbital stability of $2020 \mathrm{XL}_{5}$, we applied the same approach also to $2010 \mathrm{TK}_{7}$, the other known ET. In Fig. 6 we report the evolution of its $\lambda$ over the integration interval. In this case, the $t_{0}=0$ is set as the mean epoch of the observations arc of $2010 \mathrm{TK}_{7}$, which corresponds to 2012 August. The results obtained on the stability in $L_{4}$ are fully consistent with the ones reported in the literature ${ }^{8,24}$, confirming the validity of our computational methods and approach.

Photometry. In order to perform our photometric measurements, we optimized the object's SNR by creating different stacked images on the motion direction of $2020 \mathrm{XL}_{5}$. In order to create the stacked images and to make the photometric measurements, we used the publicly available Tycho Tracker software ${ }^{41}$. For each night, we started selecting all the frames gathered for each filter to create a stacked image and measure the object's SNR. We then repeated the process after removing the last frame ordered chronologically and we iterated the process unti we were left with only four images to stack. We then compared the SNR values obtained for the different stacks and selected the image with the target having the highest SNR. With this process, we managed to obtain the best possible photometry, despite the nearly saturated background due to the twilight proximity. We then used 3.5 pixel apertures to measure the flux of $2020 \mathrm{XL}_{5}$. As comparison stars, we selected all the solar analog stars in the field and we used the values of the ATLAS catalog ${ }^{42}$ to compute the absolute photometry for the different bands used in our observations.

In order to select the stacked images suitable for photometry, we applied a threshold filter of SNR $>5$. From the follow-up observations gathered with SOAR, we could obtain three $r^{\prime}$ measurements and two with $i^{\prime}$ and $g^{\prime}$ filters. Observations with LDT allowed us to extract a measurement in the $V$ filter. We also checked the recovery data and managed to extract two $r^{\prime}$ measurements from the Pan-STARRS data. Unfortunately, DECam's data had an SNR $<3$, which was not enough to extract any photometric measurement. The photometric measurements for the different bands and nights are presented in Table 3.

Color indices. We estimated the reflectance values at the wavelengths of standard Sloan Digital Sky Survey (SDSS) filters following a procedure from the literature ${ }^{43}$. We first computed the solar-corrected ${ }^{44}$ colors and albedos, normalized at the $r$ band, and their corresponding uncertainties.

Furthermore, we computed the $a^{*}$ parameter ${ }^{45} a^{*}=0.9285(g-r)+0.3712$ $(r-i)-0.66204$, which turns out to be $a^{*}=-0.9 \pm 0.6$ for $2020 \mathrm{XL}_{5}$ on the first observing night, and is more consistent with C-complex objects ${ }^{44}$; on the other hand, we obtained $a^{*}=-0.4 \pm 1.0$ for the second night, compatible with all 

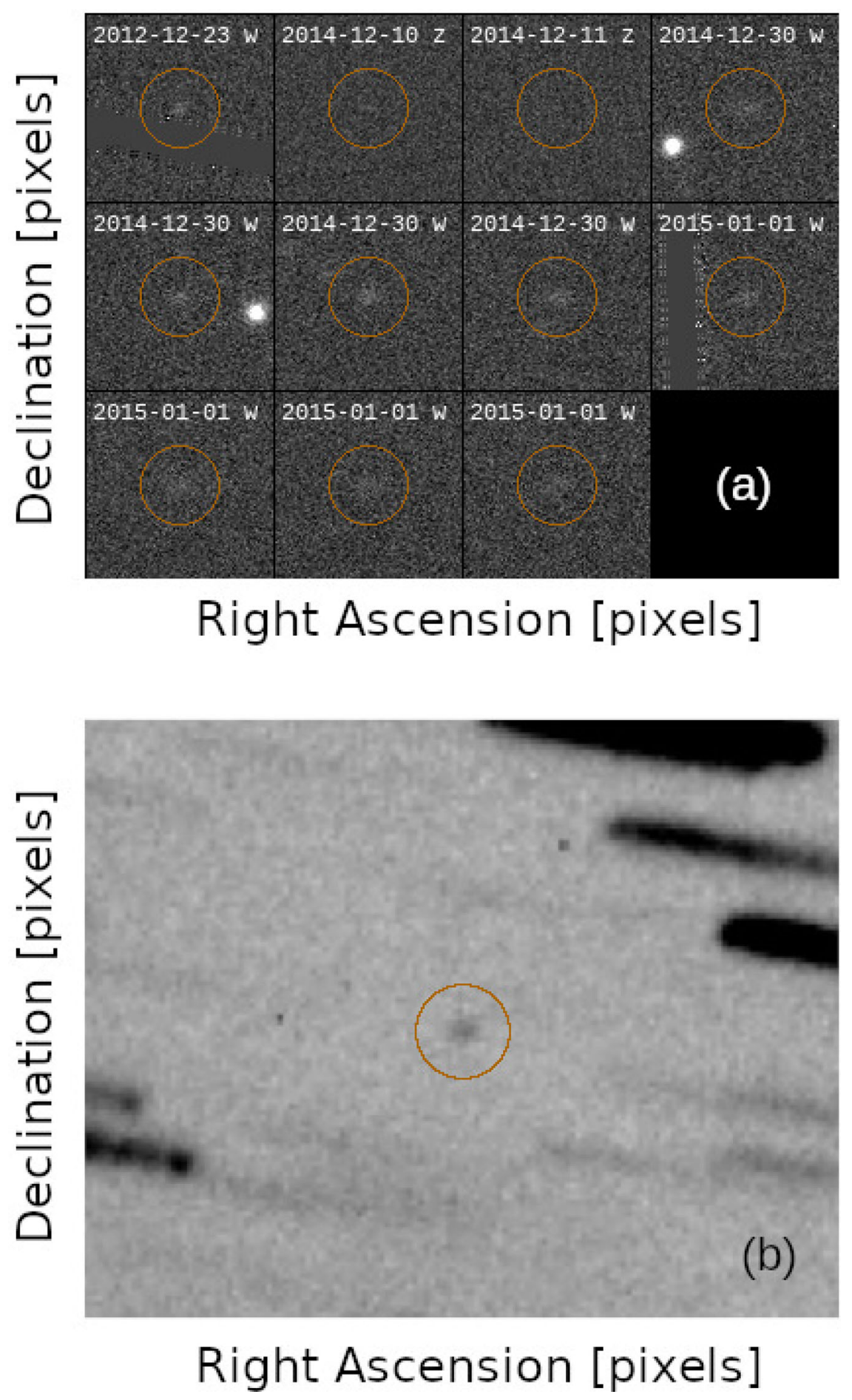

Fig. 5 Example of detections. a A mosaic showing the Pan-STARRS pre-discovery observations of $2020 X_{5}$. The orange circles highlight the position of the object. $\mathbf{b}$ Overall stack of the 13 frames obtained with the Lowell Discovery Telescope on 2021 February 22. The orange circle highlights the position of the object.

possible complexes ${ }^{44}$. Additionally, we plotted the $g-r$ and $r-i$ colors indices of $2020 \mathrm{XL}_{5}$ together with objects from the SDSS MOV catalog release 3 (see Fig. 3). From the SDSS catalogs, we selected only those objects which fulfill the quality criteria as found in the literature ${ }^{46}$. The estimated colors lie outside the space occupied by most other asteroids from the SSDS but are offset towards the $\mathrm{C}$-complex objects. The uncertainties of colors estimated from the second night are compatible with all possible taxonomic complexes.

Phase curve. We used the magnitudes in the $r$ filter to construct a phase curve for $2020 \mathrm{XL}_{5}$ (see Fig. 7). We first normalized the magnitudes to unit distances to the
Earth and the Sun according to $r(\alpha)=r(R, \Delta, \alpha)-5 \log (R \Delta)$, where $\alpha$ is the phase angle, $R$ is the heliocentric distance, and $\Delta$ the geocentric distance (both in au). We then applied the HG12* model ${ }^{47}$ using the online version of the tool ${ }^{48}$. Because of the large phase angles and the low coverage of the phase curve, the best fit we obtained assumes a single free parameter, the absolute magnitude, while $G 12^{*}$ is fixed, according to different taxonomical types. In our case, the best fit was obtained for the $\mathrm{P}$ and $\mathrm{C}$ types. The error in the magnitudes is estimated using Monte Carlo methods and the lower (upper) uncertainty corresponds to the 16th (84th) percentile. Note that the last point in the phase curve, at about $\alpha=80^{\circ}$, was obtained from the measured $V$ magnitude using the transformations from Sloan's ugriz to UBVRI ${ }^{49}$. 


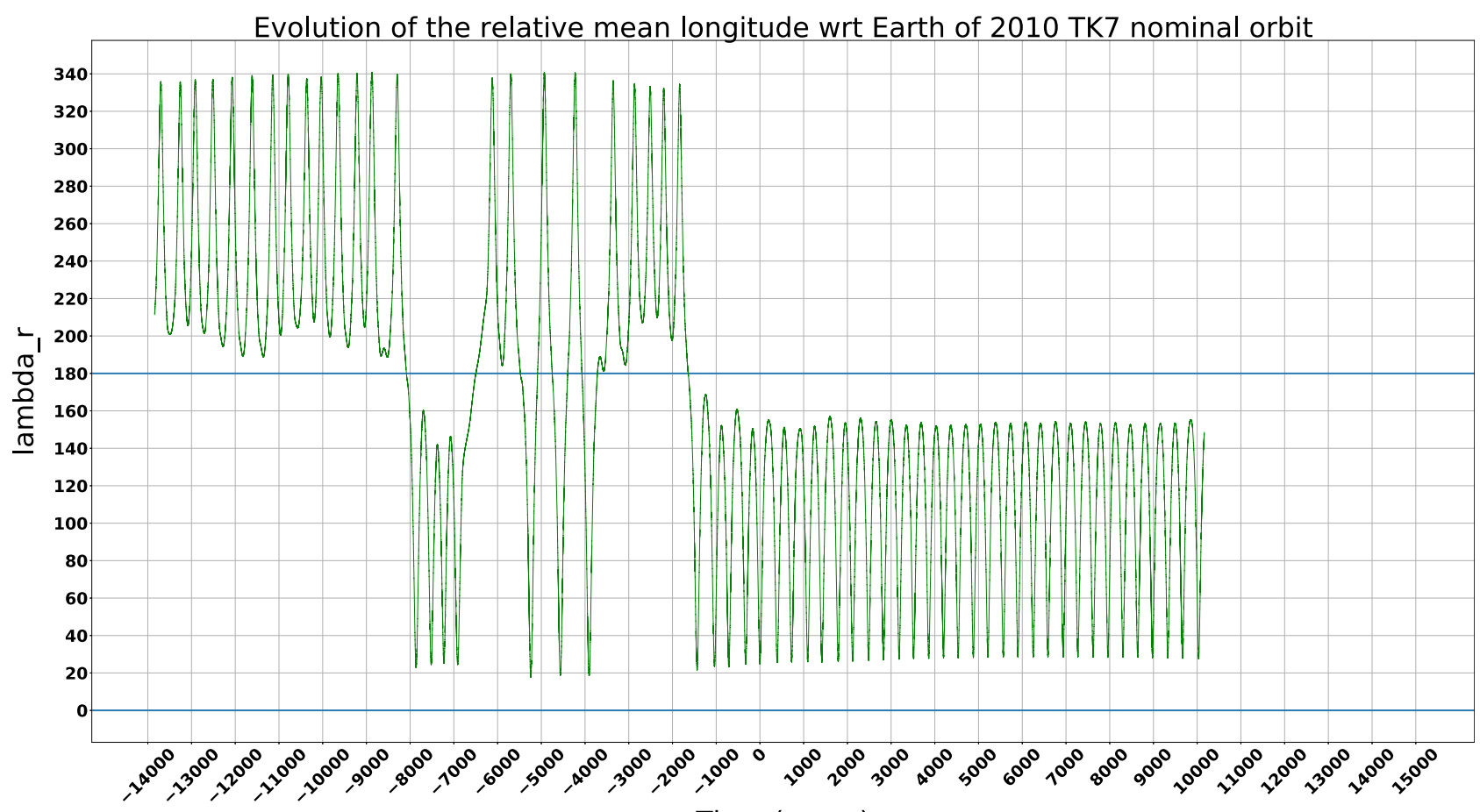

Time (years)

Fig. 6 Mean longitude evolution analysis of $\mathbf{2 0 1 0}$ TK. Evolution of $\lambda_{r}$ for the nominal orbit of $2010 \mathrm{TK}_{7}$ along 29,000 years. Source data are provided as a Source Data file.

Table 3 Photometry.

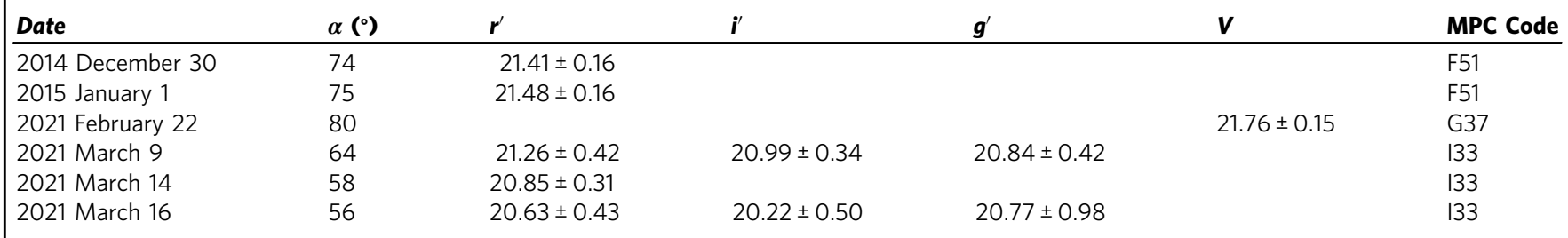

Photometric measurements of $2020 X L_{5}$ as defined in the text. The table presents the phase angle at the moment of observation and the measured photometry in $r^{\prime}, i^{\prime}, g^{\prime}$, and $V$ bands, together with the MPC code of the telescope used for the observation.

Delta-v budget: patched-conics approach. Despite the proximity of the ETs orbits, they are still deep-space targets, and thus, any practical mission to this kind of orbits is likely to make use of gravity-assists and/or low-thrust solar electric propulsion (SEP). However, for the purpose of this paper, we limited our search to sub-optimal delta-v trajectories using a patched-conics approach with ballistic trajectory.

For the heliocentric segment of the transfer trajectory, we solved Lambert's boundary value problem (BVP) in order to determine the Keplerian orbit that connects the spacecraft and the target asteroid in space in a given elapsed time. Lambert's BVP solver is very computationally fast, which allows us to do a quick survey of ballistic transfers from Earth to $2020 \mathrm{XL}_{5}$ for different departure dates and time-of-flights (TOF). For this paper, we used the multi-revolutions Lambert's problem solver ${ }^{50}$ implemented within the pykep software ${ }^{31}$ by the mission analysis team at ESA/ESOC.

For the geocentric portion of the trajectory, the escape orbit is calculated starting from both a low Earth orbit (LEO) at approximately $300 \mathrm{~km}$, and a geostationary transfer orbit (GTO) with a perigee altitude of also $300 \mathrm{~km}$ and the apogee at GEO altitude. In general, similar research works only deal with a simple launch from LEO; however, it is very expensive to go from LEO directly to escape with only chemical propulsion. For this reason, we also considered the GTO orbit, which is more common in actual space missions (especially if we assume a shared launch) and reduces significantly the cost of getting out of the Earth's sphere of influence (SOI).

Estimation of the total delta-v. The total delta-v for this mission is estimated as the sum of the delta-v required to go from launch conditions to Earth's escape orbit, $d v_{1}$, and the delta-v required to do the asteroid-rendezvous, $d v_{2}$, which is zero for a fly-by mission. For a given transfer trajectory by Lambert's problem, we can compute $d v_{1}$ as the excess velocity required at the perigee of the starting orbit in order to enter an Earth's escape trajectory with a velocity at infinity $\left(v_{\text {inf }}\right)$ equal to the relative velocity difference between the Earth and the calculated starting velocity of the transfer trajectory at the launch date. Similarly, $d v_{2}$ can be computed as the velocity difference between the target asteroid and the calculated ending velocity of the transfer trajectory at the arrival date.

For each combination of departure date and TOF, we can compute a single set of $d v_{1}$ and $d v_{2}$. Therefore, in order to find the optimal transfer trajectory, we have performed a scan in TOF, between 180 days and 3 years and a time step of 1 day, and we selected the minimum total delta-v $\left(d v_{1}+d v_{2}\right)$ as the optimal solution for the departure date considered. We have repeated this process for the next departure date, which is about 0.5 days later. The launch window considered goes from 2021 January 1 until 2026 January 1 . This time window is enough for the purposes of this study, since the results are completely periodic, and thus, similar results can be expected for other departure dates. Additionally, we have validated our results using the JPL Small-Body Mission-Design online Tool $^{51}$, which provided very similar results.

\section{Data availability}

The datasets generated during and/or analysed during the current study are available from the corresponding author on reasonable request. DECam data are available in the NOIRLab Archive Astro Data Archive (https://astroarchive.noirlab.edu). Pan-STARRS data are available upon request to R. Wainscoat (rjw@hawaii.edu). Catalina Sky Survey data are available upon request to E. Christensen (eric@lpl.arizona.edu). Lowell 


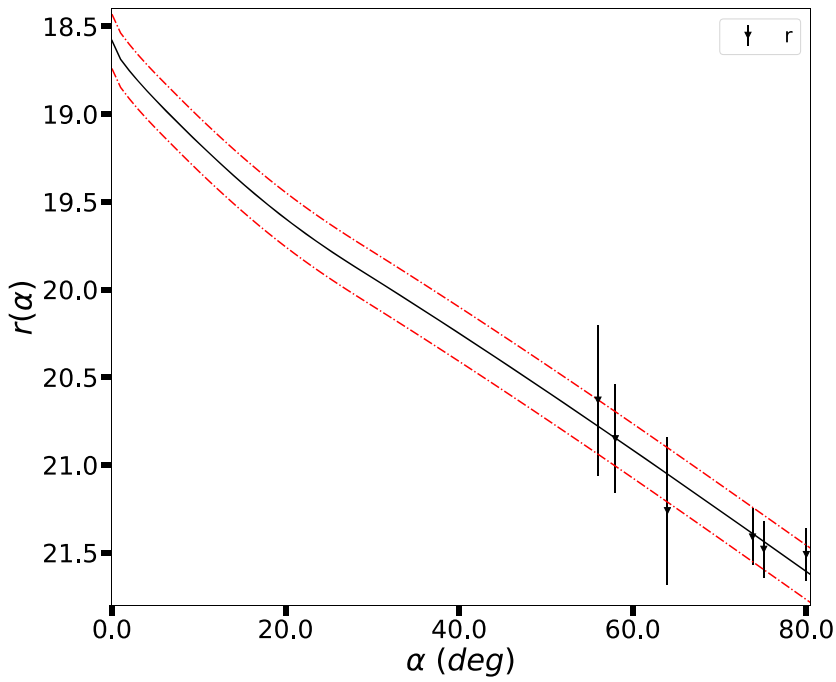

Fig. 7 Photometric phase curve. Phase curve in the $r$ filter of $2020 \mathrm{XL}_{5}$ using the six observing nights. The dashed line indicates the adopted solution, while the dot-dashed line indicates the uncertainty interval of the absolute magnitude. Source data are provided as a Source Data file.

Discovery Telescope data and SOAR data are available upon request to the corresponding author. Astrometric measurements are available on the MPC site (http:// www.minorplanetcenter.net/db_search/show_object?object_id=2020+XL5). Source data are provided with this paper.

\section{Code availability}

The ESA NEO Coordination Centre's orbit determination and impact monitoring AstOD software used for the study of the orbit stability is proprietary (https:// neo.ssa.esa.int/about-neocc). The Tycho Tracker software was used for data processing and photometric analysis (http://www.tycho-tracker.com). The pykep scientific library was used for the delta-v budget calculations (http://esa.github.io/pykep).

Received: 10 July 2021; Accepted: 21 December 2021; Published online: 01 February 2022

\section{References}

1. Szebehely, V. Theory of Orbits. The Restricted Problem of Three Bodies (Academic Press, 1967).

2. Trojan Minor Planets listed by the MPC. https://www.minorplanetcenter.net/ $\mathrm{iau} /$ lists/Trojans.html.

3. de la Fuente Marcos, C. \& de la Fuente Marcos, R. Asteroid 2013 ND $_{15}$ : Trojan companion to Venus, PHA to the Earth. Mon. Not. R. Astron. Soc. 439, 2970-2977 (2014).

4. de La Fuente Marcos, C. \& de La Fuente Marcos, R. Three new stable L5 Mars trojans. Mon. Not. R. Astron. Soc. 432, L31-L35 (2013).

5. Dvorak, R. \& Schwarz, R. On the stability regions of the Trojan asteroids. Celest. Mech. Dyn. Astron. 92, 19-28 (2005).

6. de la Fuente Marcos, C. \& de la Fuente Marcos, R. Asteroid $2015 \mathrm{DB}_{216}$ : a recurring co-orbital companion to Uranus. Mon. Not. R. Astron. Soc. 453, 1288-1296 (2015).

7. Sheppard, S. S. \& Trujillo, C. A. A thick cloud of Neptune Trojans and their colors. Science 313, 511-514 (2006).

8. Connors, M., Wiegert, P. \& Veillet, C. Earth's Trojan asteroid. Nature 475, 481-483 (2011).

9. Mikkola, S. \& Innanen, K. A. Studies on solar system dynamics. II. The stability of Earth's Trojans. Astron. J. 100, 290 (1990).

10. Brasser, R. et al. Transient co-orbital asteroids. Icarus 171, 102-109 (2004).

11. Wiegert, P. A., Innanen, K. A. \& Mikkola, S. An asteroidal companion to the Earth. Nature 387, 685-686 (1997).

12. Whiteley, R. J. \& Tholen, D. J. A CCD search for Lagrangian asteroids of the Earth-Sun system. Icarus 136, 154-167 (1998).

13. Markwardt, L. et al. Search for L5 Earth Trojans with DECam. Mon. Not. R. Astron. Soc. 492, 6105-6119 (2020).
14. Lifset, N., Golovich, N., Green, E., Armstrong, R. \& Yeager, T. A search for L4 Earth Trojan asteroids using a novel track-before-detect multiepoch pipeline. Astron. J. 161, 282 (2021)

15. Cambioni, S. et al. An Upper Limit on Earth's Trojan Asteroid Population from OSIRIS-REx. In 49th Annual Lunar and Planetary Science Conference, Lunar and Planetary Science Conference. 1149 (2018).

16. Yoshikawa, M. et al. Mission Status of Hayabusa2. In 49th Annual Lunar and Planetary Science Conference, Lunar and Planetary Science Conference. 1771 (2018).

17. Dunbar, R. S. \& Helin, E. F. Estimation of an upper limit on the Earth Trojan asteroid population from Schmidt survey plates. Bull. Am. Astron. Soc. 15, 830 (1983).

18. de la Fuente Marcos, C. \& de la Fuente Marcos, R. Transient terrestrial Trojans: comparative short-term dynamical evolution of $2010 \mathrm{TK}_{7}$ and $2020 \mathrm{XL}_{5}$. Res. Notes Am. Astronomical Soc. 5, 29 (2021).

19. ESA NEO Coordination Centre. Orbit determination and impact monitoring AstOD software at NEOCC. https://neo.ssa.esa.int/-/impact-monitoringinformation-now-computed-by-neocc (2020).

20. Hainaut, O. R. et al. Elimination of a virtual impactor of 2006 QV89 via deep non-detection. A\&A 653 (2021).

21. Milani, A. \& Gronchi, G. Theory of Orbit Determination (Cambridge Univ. Press, 2008).

22. William, M., F., James, G., W., Dale, H., B., Ryan, S., P. \& Kuchynka, P. The JPL planetary and lunar ephemerides DE430 and DE431. (2014).

23. Lagrange, J. L. Essai sur le probléme des trois corps. Oeuvres de Lagrange 6 , 229-332 (1772)

24. Dvorak, R., Lhotka, C. \& Zhou, L. The orbit of 2010 TK7: possible regions of stability for other Earth Trojan asteroids. A\&A 541 (2012).

25. Namouni, F. \& Murray, C. D. The effect of eccentricity and inclination on the motion near the Lagrangian points $\mathrm{L}_{4}$ and $\mathrm{L}_{5}$. Celest. Mech. Dyn. Astron. 76, 131-138 (2000).

26. Tsiganis, K., Dvorak, R. \& Pilat-Lohinger, E. Thersites: a 'jumping' Trojan? Astron. Astrophys. 354, 1091-1100 (2000).

27. Ivezić, Ž. et al. Color confirmation of asteroid families. Astron. J. 124, 2943 (2002).

28. Oszkiewicz, D. A. et al. Asteroid taxonomic signatures from photometric phase curves. Icarus 219, 283-296 (2012).

29. Usui, F., Hasegawa, S., Ishiguro, M., Müller, T. G. \& Ootsubo, T. A comparative study of infrared asteroid surveys: IRAS, AKARI, and WISE. Publ. Astron. Soc. Jpn. 66, 56 (2014).

30. Greg Stacey, R. \& Connors, M. Delta-v requirements for earth co-orbital rendezvous missions. Planet. Space Sci. 57, 822-829 (2009).

31. pykep scientific library. https://esa.github.io/pykep/.

32. Marzari, F. \& Scholl, H. Long term stability of Earth Trojans. Celest. Mech. Dyn. Astron. 117, 91-100 (2013).

33. Granvik, M. et al. Escape of asteroids from the main belt. Astron. Astrophys 598, A52 (2017).

34. Galiazzo, M. A. \& Schwarz, R. The Hungaria region as a possible source of Trojans and satellites in the inner Solar system. Mon. Not. R. Astron. Soc. 445 , 3999-4007 (2014).

35. DeMeo, F. E. \& Carry, B. Solar System evolution from compositional mapping of the asteroid belt. Nature 505, 629-634 (2014).

36. Levine, S. E. et al. Status and performance of the discovery channel telescope during commissioning. In Ground-based and Airborne Telescopes IV 844419 (International Society for Optics and Photonics, 2012).

37. Bida, T. A., Dunham, E. W., Massey, P. \& Roe, H. G. First-generation instrumentation for the discovery channel telescope. In Ground-based and Airborne Instrumentation for Astronomy V 91472N (International Society for Optics and Photonics, 2014).

38. T., T. N. Essentials of Monte Carlo Simulation (Springer-Verlag, New York, 2013).

39. Python3. https://www.python.org/downloads.

40. The OrbFit Software Package. http://adams.dm.unipi.it/orbfit/.

41. Tycho Tracker software. https://www.tycho-tracker.com/.

42. Tonry, J. L. et al. The ATLAS all-sky stellar reference catalog. Astrophys. J. 867, 105 (2018).

43. Roig, F. \& Gil-Hutton, R. Selecting candidate V-type asteroids from the analysis of the Sloan Digital Sky Survey colors. Icarus 183, 411-419 (2006).

44. Ivezić, Ž. et al. Solar system objects observed in the Sloan Digital Sky Survey Commissioning Data. Astron. J. 122, 2749-2784 (2001).

45. Roig, F. \& Gil-Hutton, R. Selecting candidate v-type asteroids from the analysis of the sloan digital sky survey colors. Icarus 183, 411-419 (2006).

46. DeMeo, F. \& Carry, B. The taxonomic distribution of asteroids from multifilter all-sky photometric surveys. Icarus 226, 723-741 (2013).

47. Penttilä, A., Shevchenko, V. G., Wilkman, O. \& Muinonen, K. H, G $\mathrm{G}_{1}, \mathrm{G}_{2}$ photometric phase function extended to low-accuracy data. Planet. Space Sci. 123, 117-125 (2016). 
48. Online calculator for H,G1,G2 photometric system. http://h152.it.helsinki.fi/ HG1G2.

49. Jester, S. et al. The Sloan Digital Sky Survey view of the Palomar-Green Bright Quasar Survey. Astron. J. 130, 873-895 (2005).

50. Izzo, D. Revisiting Lambert's problem. Celest. Mech. Dyn. Astron. 121, 1-15 (2015).

51. JPL's small-body mission-design tool. https://ssd.jpl.nasa.gov/tools/ mdesign.html\#/intro.

\section{Acknowledgements}

We thank Federica Spoto for her assistance on the independent check of the orbit calculations done with the OrbFit software. The work of TS-R was carried out through grant APOSTD/2019/046 by Generalitat Valenciana (Spain). This work was (partially) funded by the Spanish MICIN/AEI/10.13039/501100011033 and by "ERDF A way of making Europe" by the "European Union" through grant RTI2018-095076-B-C21, and the Institute of Cosmos Sciences University of Barcelona (ICCUB, Unidad de Excelencia 'María de Maeztu') through grant CEX2019-000918-M. P-YL acknowledges NEO-MAPP project under H2020-SPACE-2019 GA 870377. PGB, and ACB acknowledge funding from the Spanish MICINN project RTI2018-099464-B-I00. AA-C acknowledges support from the State Agency for Research of the Spanish MCIU through the "Center of Excellence Severo Ochoa” award to the Instituto de Astrofísica de Andalucía (SEV-20170709). DO was supported by National Science Centre, Poland, grants numbers 2017/26/ D/ST9/00240 and 2017/25/B/ST9/00740. Based on observations obtained at the Southern Astrophysical Research (SOAR) telescope, which is a joint project of the Ministério da Ciência, Tecnologia e Inovações (MCTI/LNA) do Brasil, the US National Science Foundation's NOIRLab, the University of North Carolina at Chapel Hill (UNC), and Michigan State University (MSU). The Catalina Sky Survey is funded by NASA's Planetary Defense Coordination Office, under grant 80NSSC18K1130. The Joan Oró Telescope (TJO) of the Montsec Observatory (OdM) is owned by the Catalan Government and operated by the Institute for Space Studies of Catalonia (IEEC). MD acknowledges funding from NASA NEOO grant 80NSSC21K0045 in support of the Second Lunation NEO Follow-up. These results made use of the Lowell Discovery Telescope (LDT) at Lowell Observatory. Lowell is a private, non-profit institution dedicated to astrophysical research and public appreciation of astronomy and operates the LDT in partnership with Boston University, the University of Maryland, the University of Toledo, Northern Arizona University, and Yale University. The Large Monolithic Imager (LMI) was built by Lowell Observatory using funds provided by the National Science Foundation (AST-1005313). Operation of the Pan-STARRS telescope is supported by the National Aeronautics and Space Administration under Grant No. 80NSSC18K0971 issued through the SSO Near-Earth Object Observations Program. This project used public archival data from the Dark Energy Survey (DES) as distributed by the Astro Data Archive at NSF's NOIRLab. Funding for the DES Projects has been provided by the DOE and NSF (USA), MISE (Spain), STFC (UK), HEFCE (UK), NCSA (UIUC), KICP (U. Chicago), CCAPP (Ohio State), MIFPA (Texas A\&M), CNPQ, FAPERJ, FINEP (Brazil), MINECO (Spain), DFG (Germany), and the collaborating institutions in the Dark Energy Survey, which are Argonne Lab, UC Santa Cruz, University of Cambridge, CIEMAT-Madrid, University of Chicago, University College London, DESBrazil Consortium, University of Edinburgh, ETH Zürich, Fermilab, University of Illinois, ICE (IEECCSIC), IFAE Barcelona, Lawrence Berkeley Lab, LMU München and the associated Excellence Cluster Universe, University of Michigan, NOIRLab, University of Nottingham, Ohio State University, OzDES Membership Consortium, University of Pennsylvania, University of Portsmouth, SLAC National Lab, Stanford University, University of Sussex, and Texas A\&M University. Based on observations at Cerro Tololo Inter-American Observatory, a program of NOIRLab
(NOIRLab Prop. ID 2012B-0001; PI: J. Frieman), which is managed by the Association of Universities for Research in Astronomy (AURA) under a cooperative agreement with the National Science Foundation. This research has made use of data and/or services provided by the International Astronomical Union's Minor Planet Center.

\section{Author contributions}

T.S.-R. coordinated the observation campaign, contributed to the writing of the proposals, performed the photometric measurements, and participated in all aspects of the discussion and to the paper writing; M.M. contributed to the writing of the proposals, performed the astrometric measurements, and participated in all aspects of the discussion and to the paper writing; L.Fa. and R.C. performed the orbit stability analysis, participated to the discussion of the paper results, and contributed to the paper writing; M.D. contributed to the writing of the proposals, performed observations, and contributed to the paper writing; A.A.-C. contributed to the writing of the proposals, performed the phase curve analysis, and contributed to the paper writing; D.O. perform the color indices analysis and contributed to the paper writing; O.R. and P.-Y.L. performed the delta-v budget analysis and contributed to the paper writing; P.G.B. and A.C.B. participated to the discussion. C.B. and L.Fr. carried out observations at SOAR. R.W. measured the PS data and participated in the discussion. E.J.C., R.J.W., and L.C. provided access to data archives and participated in the discussion. All authors reviewed the manuscript.

\section{Competing interests}

The authors declare no competing interests.

\section{Additional information}

Supplementary information The online version contains supplementary material available at https://doi.org/10.1038/s41467-022-27988-4.

Correspondence and requests for materials should be addressed to T. Santana-Ros.

Peer review information Nature Communications thanks the anonymous reviewers for their contribution to the peer review of this work.

Reprints and permission information is available at http://www.nature.com/reprints

Publisher's note Springer Nature remains neutral with regard to jurisdictional claims in published maps and institutional affiliations.

(i) Open Access This article is licensed under a Creative Commons Attribution 4.0 International License, which permits use, sharing, adaptation, distribution and reproduction in any medium or format, as long as you give appropriate credit to the original author(s) and the source, provide a link to the Creative Commons license, and indicate if changes were made. The images or other third party material in this article are included in the article's Creative Commons license, unless indicated otherwise in a credit line to the material. If material is not included in the article's Creative Commons license and your intended use is not permitted by statutory regulation or exceeds the permitted use, you will need to obtain permission directly from the copyright holder. To view a copy of this license, visit http://creativecommons.org/ licenses/by/4.0/.

(c) The Author(s) 2022 